\title{
A Service Evaluation Project Comparing Telephone and Face to Face Colorectal 2 Week Wait Clinics
}

\section{Karen A Cock ${ }^{*}$}

Department of Outpatients, Royal Cornwall Hospital NHS Trust, Plymouth University, Cornwall, UK

*Corresponding author: Karen A Cock, Department of Outpatients, Royal Cornwall Hospital NHS Trust, Plymouth University, Cornwall, UK, Tel: 07791657470; E-mail: karen.cock@rcht.cornwall.nhs.uk

Received date: February 20, 2018; Accepted date: February 24, 2018; Published date: March 1, 2018

Copyright: (C) 2018 Cock KA. This is an open-access article distributed under the terms of the Creative Commons Attribution License, which permits unrestricted use, distribution, and reproduction in any medium, provided the original author and source are credited.

\begin{abstract}
Aim: To determine if a virtual (telephone) two week wait (2 ww) clinic affects patient satisfaction and delivers a comparable service to a traditional face to face assessment.

Study design: Part one is a case cohort comparative study of 100 consecutive patients (divided by referral criteria into virtual $(n=50)$ and face to face $(n=50)$ cohorts). Patient satisfaction in both cohorts was assessed by an adapted version of the Grogan et al. validated patient satisfaction questionnaire. The questionnaire was piloted first and was found to have high internal reliability (Cronbach's alpha=0.945). Part two is a three month data capture to compare specific quality indicators, such as, number of referrals for additional investigations or further clinic episodes between the two cohorts (virtual $n=251$ and face to face $n=403$ ).
\end{abstract}

Results: Overall satisfaction scores showed; $90 \%$ of patients in the virtual cohort and $98 \%$ of patients in the face to face cohort, strongly agreed that they were satisfied with the care they received. Mean satisfaction scores in the two cohorts revealed that the virtual cohort achieved less strongly agrees than the face to face cohort but this was not significant $(p=0.5)$. Further analysis of the responses to each question demonstrated no statistical significance when comparing the two cohorts. Analysis of the three month data collection revealed no significant difference between the two groups and referrals for additional investigations (virtual $10 \%, n=25$. Face to face $9.4 \% n=38$ ) However, the face to face group yielded a higher number of further clinic episodes (virtual $1 \%, n=1$. Face to face $3 \%$, $n=16$ ) and colorectal cancer diagnoses (virtual $4 \%, n=10$. Face to face $10 \%, n=40$ ).

Conclusions: The study offered patients the opportunity to reflect on service delivery enabling a more responsive approach to healthcare within the colorectal 2 ww service. It provides strong evidence that virtual 2 ww clinics provide an assessment method that is essentially, as acceptable as traditional face to face clinics.

Keywords: Colorectal 2 week wait; Patient satisfaction; Virtual 2 week wait clinics; Validated satisfaction questionnaire

\section{Introduction}

\section{Background}

In the United Kingdom (UK) more than 40,000 people are diagnosed with bowel cancer every year, equating to one person every 13 minutes [1]. The prevalence of this disease resulted in the development of quick access clinics known as two week wait (2 ww). Patients displaying any of the following five symptoms (Table 1) for six weeks or longer meet the $2 \mathrm{ww}$ criteria and warrant referral to a team specialising in the management of lower gastrointestinal cancer [2]. The implementation of the colorectal $2 \mathrm{ww}$ guideline in 2000 dramatically increased the need for service provision, leading to many hospital trusts throughout the country utilising specialist nurses to increase their capacity to meet this demand. This was further compounded by a broadening of the guideline in 2015 (Table 2) to reduce the cancer detection rate from $7-3 \%$ which resulted in referrals at the authors district general hospital (DGH) expanding from approximately 1500 to 3500 per year [3]. The upsurge in referrals led to an innovative approach to 2 ww service delivery and the creation of a virtual side to the traditional $2 \mathrm{ww}$ colorectal clinics.

\begin{tabular}{|l|l|}
\hline 1 & $>40$ years of age with rectal bleeding and change in bowel habit \\
\hline 2 & $>60$ years of age with rectal bleeding without change in bowel habit \\
\hline 3 & $>60$ years of age with change in bowel habit \\
\hline 4 & Palpable rectal mass not dependent on age \\
\hline 5 & Lower abdominal mass consistent with involvement of the large bowel \\
\hline
\end{tabular}




\begin{tabular}{|l|l|}
\hline 6 & $>40$ years of age with unexplained weight loss and abdominal pain. \\
\hline 7 & $<50$ with rectal bleeding and any of the following unexplained symptoms: Weight loss, change in bowel habit, abdominal pain or iron deficiency anaemia. \\
\hline
\end{tabular}

Table 1: Colorectal symptoms warranting 2 ww referral and additional criteria.

\section{Study Aims}

To determine if a virtual (telephone) two week wait (2 ww) clinic affects patient satisfaction and delivers a comparable service to a traditional face to face assessment.

\section{Study design}

The setting for the study was an acute teaching hospital that provides care for approximately 500,000 people living across the county. Details of any patient referred under the 2 ww guideline were faxed to the hospital using a standard pro forma. Patients were then allocated to be seen in the clinic within the two-week timeframe.

Throughout the study period clinics were performed by four colorectal specialist nurses and two doctors. All clinicians underwent the same training to be qualified in 2 ww clinic delivery, followed the same strict protocol during the consultations and all appointments were allocated the same duration of time.

Part one: All patients referred to the 2 ww clinic during the study period were invited to take part anonymously and confidentiality was ensured. Participants were allocated to the respective cohorts (virtual or face to face) by the $2 \mathrm{ww}$ booking office staff, the booking team randomised patients into the cohorts based on their referral criteria selected by the referring general practitioner (Table 2). All participants that have either a mass or are $>80$ years of age are included in the face to face group to enable examination in clinic and more accurate clinical assessment of general fitness for investigations. The rationale for this age group being seen in clinic is the anticipated increase in comorbidities associated with age [4].

\begin{tabular}{|l|l|l|l|l|l|}
\hline Symptoms & \multicolumn{4}{l|}{ Patient age } & \multicolumn{4}{l|}{} \\
\hline- & Under 50 & Over 40 & 50 to 60 & 60 to 80 & Over 80 \\
\hline Looser and more frequent motions for $>3$ weeks & - & - & - & PHONE & CLINIC \\
\hline Unexplained Rectal bleeding & - & - & PHONE & PHONE & CLINIC \\
\hline Unexplained Iron deficiency anaemia & - & - & PHONE & PHONE & CLINIC \\
\hline Rectal or abdominal mass & - & - & CLINIC & CLINIC & CLINIC \\
\hline Positive faecal occult blood (FOB) test & - & - & CLINIC & CLINIC \\
\hline Rectal bleeding and anaemia & PHONE & - & - & - & - \\
\hline Rectal bleeding and change in bowels & PHONE & & - & - & - \\
\hline Rectal bleeding and abdominal pain & CLINIC & - & - & - & - \\
\hline Rectal bleeding and weight loss & CLINIC & - & - & - & - \\
\hline Unexplained anal mass or anal ulceration & CLINIC & CLINIC & CLINIC & CLINIC & CLINIC \\
\hline Unexplained weight loss and abdominal pain & - & CLINIC & CLINIC & CLINIC & CLINIC \\
\hline
\end{tabular}

Table 2: Referral criteria.

A week after the clinic appointments an independent team of specialist nurses (who were not involved in clinic provision) phoned each participant and invited them to take part in the study. They were then read the 10 questions over the phone and their responses recorded.

Part two: Data was collected for all patients referred through the 2 ww clinic between $1^{\text {st }}$ June and $31^{\text {st }}$ August 2017 with regards the type of investigation requested, number of additional investigations undertaken, number of additional consultant clinics and diagnosis. Data was collated by the same team of independent specialist nurses and recorded on a excel spreadsheet for analysis.

\section{Sampling}

The aim of the sampling was to capture only those people who attended the $2 \mathrm{ww}$ clinic in order to gain insight into their particular experience, thereby necessitating a convenience sampling approach. Patient satisfaction studies by Cock and Kent, Hill et al. and Grogan et al. all utilised convenience samples [5-7]. This is a non-probability sampling technique where participants are selected, not for their representativeness, but for their accessibility, enabling the researcher to answer the research question posed [8]. 


\section{Data collection tool}

The design of this study mirrors those conducted by Drewery et al., Williams et al. and Cock and Kent, all of which investigated patient satisfaction using questionnaires as their method for data collection $[5,9,10]$. Furthermore, the questionnaire used in this study was adapted from the Grogan et al. patient satisfaction questionnaire (PSQ) improving reliability and internal consistency. Permission from Grogan et al. to use and modify the PSQ was granted prior to commencement of the original study. The original PSQ is a 46 item questionnaire that examines patients' perceptions of five different dimensions: the doctor, the nurse, facilities, access and appointments. The focus of this study centred on how the patient perceived their care during the appointment and therefore the other domains were removed leaving just 10 remaining questions (Table 3). Additional information was also included: gender, age, if the appointment was on time, or if late, length of wait.

\begin{tabular}{|l|l|}
\hline Q1 & I am very satisfied with the overall care I received during my consultation \\
\hline Q2 & The health professional clearly explained examination and tests \\
\hline Q3 & I was dissatisfied with the way I was treated by the health professional \\
\hline Q4 & During my consultation I was allowed to say everything that I felt was important \\
\hline Q5 & The health professional had a genuine interest in me as a person \\
\hline Q6 & I was not given enough information by the health professional today \\
\hline Q7 & I felt that the health professional was skilled and knowledgeable about my condition \\
\hline Q8 & I was satisfied with the way I was treated by the health professional \\
\hline Q9 & The health professional made me feel I was wasting his/her time \\
\hline Q10 & I felt rushed during my consultation \\
\hline
\end{tabular}

Table 3: Ten questions.

Studies by Karthikeyan et al. and Mussard et al. used validated questionnaires to measure patient satisfaction in a variety of different settings, modifying the original tool to ensure that it was applicable to the new setting $[11,12]$. Karthikeyan et al. acknowledge the risks of adapting a validated tool and argue that checking the reliability of the modified tool dramatically reduces the associated risks.

In the study by Grogan et al. the questions were constructed from individual statements derived from interviews with patients, ensuring they were phrased in ways that would make sense to the patient. Responses were coded 1-5 from strongly-agree to strongly-disagree and both positively and negatively worded questions were used to increase rigour. Negatively worded questions were reverse scored (1=strongly-disagree, and so on) so that, at all times, low scores equalled higher satisfaction.

In addition to the 10 questions a free text box was included to enable participants to express any further views regarding their experience during the consultation. The advantage of including an open ended question is that it provides respondents with the freedom to answer the question in their own way, unrestricted by the choices provided by the researcher. Permitting self-expression and a richness in detail not possible with purely closed questions [13].

The modified questionnaire used within this study was previously assessed for internal reliability using Cronbach's alpha. The data generated a Cronbach's alpha coefficient of 0.945 demonstrating that the questionnaire maintained a high level of internal reliability [5].

Additionally, for the second part of the study the specialist nurses accessed the $2 \mathrm{ww}$ database and electronic patient records to identify consecutive patients during the three month study period, enabling them to record the number of investigations, quantity of appointments and diagnoses for each patient.

\section{Relevance to practice}

The positive views obtained from patients', support the utilisation of a virtual (telephone) approach for some referral criteria to colorectal 2 ww clinics providing a responsive way to provide clinics which may encourage other specialities to utilise this type of framework.

\section{Pilot Study}

A pilot study utilising the modified questionnaire was conducted during a previous study undertaken by the author to compare patient satisfaction between nurse-led and doctor-led colorectal $2 \mathrm{ww}$ clinics in order to address some of the disadvantages of using a modified questionnaire [5]. This enabled adjustments to be made and increased rigour, enabling the questionnaire to be tested within the study setting to ensure that it was appropriate to the intended patient group [14].

\section{Data analysis}

During part one of the study, the scores for the 10 questions were summated to give an overall level of satisfaction for the two different cohorts (virtual and face to face), with the mode, median and mean then calculated. Distribution of the scores was also examined to check if patients were using the full range of possible scores and not giving uniformly strongly agree or strongly disagree responses. Statistical analysis was conducted by using a Mann-Whitney $U$ test, since the data compiled collectively from the Likert scale were ordinal in nature. The Mann-Whitney $U$ test is a non-parametric test that allows two groups to be compared without making assumptions that the data is normally distributed. It is the test of choice for this type of data [15]. The data related to total satisfaction were assessed for normality using Kolmogrov-Smirnov and Shapiro-Wilk tests. Skewness was found to be 0.242 with 20.843 Kurtosis (mean 1.070, median 1, mode 1). This 
Page 4 of 7

demonstrated that the data were not normally distributed (nonparametric) and were skewed towards the left, indicating a higher proportion of responses that are satisfied compared to dissatisfied. Consequently the use of non-parametric testing was further supported. Finally, analysis of the data recorded by the free text question was performed through thematic analysis. All responses were listed, recurrent answers were then grouped to enable identification of the overarching themes which were then analysed to determine how these themes support the quantitative analysis [16]. During part two of the study, data from consecutive patients in both the virtual and face to face cohorts over a three month period were compiled to identify.

- Total number of investigations

- Type of investigation booked

- Number of follow up clinic appointments

- Diagnosis

\section{Results}

Total number of colorectal $2 \mathrm{ww}$ patients within the first part of the study period was 100 (50 in virtual clinic and 50 in face to face clinic).

\section{Patient demographics}

Demographic data for the two different cohorts are presented in Table 4.

\begin{tabular}{|c|c|c|c|c|c|}
\hline \multicolumn{2}{|c|}{ Statistics } & \multicolumn{2}{|l|}{ TNT } & \multicolumn{2}{|l|}{$F 2 F$} \\
\hline & & Number & $\%$ & Number & $\%$ \\
\hline \multirow{2}{*}{ Gender } & Female & 20 & $40 \%$ & 26 & $52 \%$ \\
\hline & Male & 30 & $60 \%$ & 24 & $48 \%$ \\
\hline \multirow{7}{*}{ Age } & $30-40$ & 1 & $2 \%$ & 0 & 0 \\
\hline & $41-50$ & 2 & $4 \%$ & 4 & $8 \%$ \\
\hline & $51-60$ & 7 & $14 \%$ & 5 & $10 \%$ \\
\hline & $61-70$ & 20 & $40 \%$ & 5 & $10 \%$ \\
\hline & $71-80$ & 20 & $40 \%$ & 9 & $18 \%$ \\
\hline & $81-90$ & 0 & 0 & 26 & $52 \%$ \\
\hline & $91-100$ & 0 & 0 & 0 & 0 \\
\hline \multicolumn{2}{|c|}{ Total Respondents } & 50 & - & 50 & - \\
\hline
\end{tabular}

Table 4: Demographic data.
As you would expect the face to face cohort includes an older population of participants because of the referral criteria (Table 2).

\section{Patient satisfaction}

Patients were asked how strongly they agreed with the statement, 'I am very satisfied with the overall care I received during my consultation' and the responses are summarised in Figure 1.

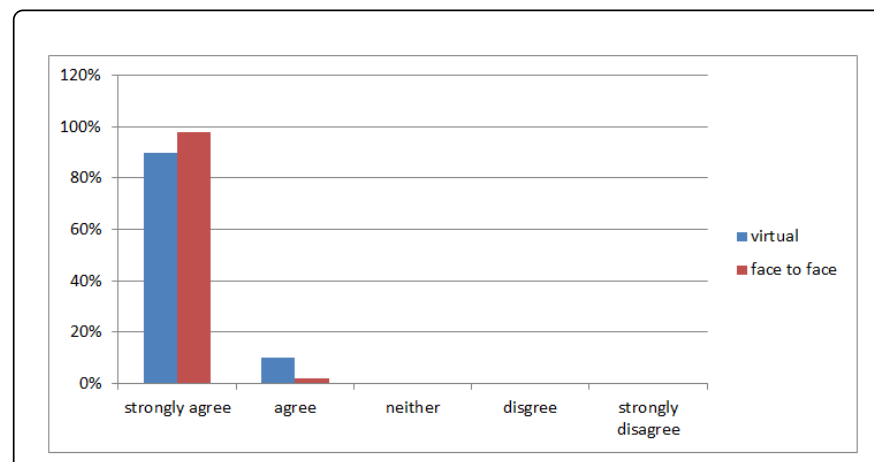

Figure 1: Illustrates how strongly the patients agreed they were satisfied overall.

All scores were summated to give an overall satisfaction score and the mean scores generated were $1.020(\mathrm{SD}=0.319$, median $=1$ and mode $=1)$ and $1.100(\mathrm{SD}=0.303$, median $=1$ and mode=1) for the virtual and face to face cohorts respectively (a score of 1 equates to the patients' strongly agreeing that they were satisfied).

Overall, results from both cohorts revealed that $94 \%$ of patients assessed through the $2 \mathrm{ww}$ clinics strongly agreed and 6\% agreed that they were very satisfied with the care they received, demonstrating that patients view the clinic positively. The difference in distribution between the two cohorts was assessed for significance using the MannWhitney $U$ test $(U=1152.5, p=0.5)$, indicating that although more strongly agreed responses were expressed by patients in the face to face cohort (virtual $n=45$, face to face $n=49$ ) this was not statistically significant.

\section{Analysis of the 10 questions}

In order to elicit areas of specific satisfaction and dissatisfaction, each of the 10 questions that are featured in the questionnaire, were analysed in turn and comparisons made between the cohorts using a Mann Whitney U test (Table 5). Threshold for significance was $\mathrm{p}=0.05$.

\begin{tabular}{|c|c|c|c|}
\hline S. No & Question & Mann-Whitney U & $P$ value \\
\hline 1 & I am very satisfied with the overall care I received during my consultation & 1152.5 & 0.502 \\
\hline 2 & The health professional clearly explained examinations and tests & 1150.5 & 0.4965 \\
\hline 3 & I was dissatisfied with the way I was treated by the health professional & 1225 & 0.8650 \\
\hline 4 & During my consultation I was allowed to say everything that I felt was important & 1152.5 & 0.502 \\
\hline 5 & The health professional had a genuine interest in me as a person & 1232 & 0.9044 \\
\hline
\end{tabular}




\begin{tabular}{|l|l|l|l|}
\hline 6 & I was not given enough information by the health professional today & 0.3125 & 1102.5 \\
\hline 7 & I felt that the health professional was skilled and knowledgeable about my condition & 0.7565 & 1204 \\
\hline 8 & I was satisfied with the way I was treated by the health professional & 0.610 & 1175 \\
\hline 9 & The health professional made me feel I was wasting his/her time & 2500 & 1 \\
\hline 10 & I felt rushed during my consultation & 1250 & 1 \\
\hline
\end{tabular}

Table 5: Comparisons for questions 1-10.

For each of the questions, the face to face cohort included more strongly agree responses to the positively worded questions and more strongly disagree responses to the negatively worded questions than the virtual cohort. This would suggest that patients in the face to face cohort were more satisfied with their experience. However, in every aspect the result was not statistically significant and therefore the virtual clinic is essentially, as acceptable as traditional face to face clinics.

\section{Analysis of free text question}

83 patients responded $(83 / 100,83 \%)$ to the free text question 'any further comments', and were comprised from both the virtual $(n=45)$ and face to face $(n=38)$ cohorts. Thematic analysis was performed on all 83 responses and the majority of responses were positive in nature.

Participants' comments included, 'This was an excellent service', 'very impressed with the service' and 'Pleased with everything, start to finish'. There were four overarching themes which emerged from the analysis:
- Clinician behaviour

- Good service

- Communication

- Being put at ease

These four themes are found throughout the literature investigating patient experience and satisfaction. Studies by Hill et al., Campbell et al. and Drewery et al. all feature several, if not all, of the above themes when identifying areas which affect patient satisfaction $[6,9,17]$. Additionally, fifteen patients (30\%) in the virtual cohort specifically mentioned that the convenience of a telephone assessment was positively received. Conversely, six patients $(12 \%)$ in the face to face cohort reported the lateness of the clinic as having a negative impact. Data from the second part of the study was also analysed and demonstrated that $89.5 \%$ of patients in the virtual cohort were booked to have endoscopic examination; the remaining $9 \%$ were booked to have radiological investigation compared to the face to face cohort which was divided into $64.5 \%$ and $31 \%$ respectively (Table 6).

\begin{tabular}{|l|l|l|l|}
\hline \multicolumn{2}{|l|}{ Statistics } & Face to face (n) & Virtual (n) \\
\hline Distribution & Total number of patients & 403 & 251 \\
\hline & Colonoscopy & $41 \%(164)$ & $56 \%(141)$ \\
\cline { 2 - 4 } & Flexible sigmoidoscopy & $7 \%(27)$ & $7.5 \%(19)$ \\
\cline { 2 - 4 } & Upper and lower endoscopy & $16.5 \%(67)$ & $26 \%(65)$ \\
\cline { 2 - 4 } & CT Pneumocolon & $13 \%(53)$ & $5 \%(12)$ \\
\cline { 2 - 4 } & CT Enema & $18 \%(74)$ & $4 \%(10)$ \\
\hline "There is a 0.5\% discrepancy for patients who were not booked for any investigation or declined to proceed with the test & \\
\hline
\end{tabular}

Table 6: Investigations booked per cohort.

The two cohorts yielded a similar quantity of patients who required a second investigation (virtual $10 \%, \mathrm{n}=25$. Face to face $9.4 \%, \mathrm{n}=38$ ). However, there was a slight increase in the number of patients who required an additional clinic appointment with a consultant in the face to face cohort $(4 \%, \mathrm{n}=16)$ compared to the virtual cohort $(1 \%, \mathrm{n}=3)$. When comparing the diagnosis categories in the two cohorts (Table 7) the main difference is in the quantity of colorectal cancers identified with $10 \%(n=40)$ of patients in the face to face cohort being diagnosed with a colorectal cancer, compared to $4 \%(n=10)$ in the virtual cohort.

\begin{tabular}{|l|l|l|}
\hline Statistics & Face to face (n) & Virtual (n) \\
\hline Cancer & $10 \%(40)$ & $4 \%(10)$ \\
\hline
\end{tabular}

\begin{tabular}{|l|l|l|}
\hline Haemorrhoids & $7.2 \%(29)$ & $9.6 \%(24)$ \\
\hline Inflammatory bowel disease & $2.7 \%(11)$ & $2.4 \%(6)$ \\
\hline Polyps & $16.1 \%(65)$ & $23 \%(58)$ \\
\hline Diverticular disease & $30.8 \%(124)$ & $35.9 \%(90)$ \\
\hline Non colorectal cancer & $1 \%(4)$ & $0.4 \%(1)$ \\
\hline No abnormalities & $32.2 \%(130)$ & $24.7 \%(62)$ \\
\hline
\end{tabular}

Table 7: Diagnosis per cohort. 
Similarly, the percentage of extra-colonic cancers is also higher in the face to face cohort ( $1 \%, \mathrm{n}=4$ face to face. $0.4 \%$. $\mathrm{n}=1$ virtual). Conversely, there is a higher yield of polyps in the virtual cohort $(16.1 \%, n=65$ face to face. $23 \%, n=58$ virtual).

\section{Discussion}

Analysis of the 10 questions provides evidence to suggest that patient satisfaction is not significantly affected by the mode of delivering the $2 \mathrm{ww}$ clinic. These findings are supported by Connor et al., who found virtual clinics to generate patient benefits which include improved satisfaction and a reduction in journeys to hospital [18]. Furthermore, Casey et al suggest that virtual clinics are well received by patients in high volume nurse-led services as an innovative service development, achieving high satisfaction due to the convenience of the appointments [19]. This is further supported by the thematic analysis within this study.

Within part two of the study, a possible explanation for the higher percentage of patients in the virtual cohort being booked for endoscopic investigation is the younger population. The risks associated with endoscopy increase with age and tend to be restricted to those patients who are $<80$ years old [20]. Similarly, the higher number of additional clinics in the face to face group could again be due to the age of the cohort and the increase in comorbidities thus associated. Decisions regarding investigations and outcomes in this cohort are therefore more complex, requiring oversight from the consultants.

Additionally, a possible cause for the higher yield of colorectal cancer in the face to face group may be a result of the referral criteria streamlining all patients with masses into this cohort. Furthermore, the higher referral rate in the virtual cohort to endoscopic investigation explains the increased yield of polyps and lower diagnosis of extracolonic pathology. This is due to endoscopic investigations only viewing inside the colon compared to radiological imaging, which also visualises the organs outside of the colon.

\section{Limitations of the Study}

The adaptation of a validated questionnaire for data collection is a limitation to the study design. In an attempt to reduce the effects of modifying the tool a pilot study was utilised and internal reliability was assessed by performing a Cronbach's alpha coefficient, mirroring studies by Karthikeyan et al. and Mussard et al. However, further examination of the modified questionnaire utilising confirmatory factor analysis, test-retest or Bland-Altman plots would have further strengthened the results and conclusions drawn from this study [7]. Alongside this there may have been other confounding variables that the author was unaware of that may have affected patient satisfaction, such as, previous patient experiences.

A further limitation of the study is that random allocation to the cohorts was not permitted; this would have reduced bias and strengthened the findings of the study [21]. However, due to patient safety it was not possible to allocate the patients blindly into the two different cohorts. The presence of a mass or patients in an older demographic required a thorough physical examination to ensure that the correct investigation is safely requested $[4,20]$.

\section{Conclusion}

Within this study, an attempt was made to determine if a virtual (telephone) two week wait ( $2 \mathrm{ww}$ ) clinic affects patient satisfaction and delivers a comparable service to a traditional face to face assessment. The clinical outcomes or possibility for adverse events between the two cohorts were not found to be significantly different, and all clinicians received the same training in $2 \mathrm{ww}$ clinic delivery and followed the same protocol for ordering investigations. Therefore the safety and effectiveness of patients in both cohorts appeared the same.

The results of the statistical analyses found that traditional face to face clinics achieved more strongly agree responses (in the positively worded questions) and more strongly disagree responses (in the negatively worded questions) when compared to virtual clinics for this group of patients. However, it was not statistically significant and therefore these results suggest that virtual clinics are as acceptable as face to face clinics in terms of patient satisfaction.

Furthermore, although differences in key indicators, such as, type of investigation booked, number of follow up clinic appointments and diagnosis did exist, this is mostly likely due to the case cohort nature of the study and the distribution of the referral criteria between the two cohorts.

This study has offered both staff and patients the opportunity to reflect on colorectal $2 \mathrm{ww}$ service delivery at the author's DGH and to influence future care patients receive, thus enabling a more responsive approach to healthcare in this speciality. This study has provided justification for utilising a combined face to face and virtual approach to colorectal $2 \mathrm{ww}$ clinics.

\section{References}

1. Office of National Statistics (2012) Cancer Registration Statistics, England.

2. NICE (2005) Improving outcomes in colorectal cancer, Cambridge: The National Institute for Health and Care Excellence.

3. NICE (2015) Suspected cancer: Recognition and referral, Cambridge: The National Institute for Health and Care Excellence.

4. Piccirillo JF, Vlahiotis A, Barrett LB, Flood KL, Spitznagel EL, et al. (2009) The changing prevalence of comorbidities across the age spectrum. Crit Rev Oncol Hematol 67: 124-132.

5. Cock K, Kent B (2017) Patient satisfaction with clinicains in colorectal 2week wait clinics. Br J Nurs 26: 319-323.

6. Hill J, Thorpe R, Bird H (2003) Outcomes for patients with RA: A rheumatology nurse practitioner clinic compared to standard outpatient care. Musculoskeletal care 1: 5-20.

7. Grogan S, Connor M, Norman P, Willits D, Porter I (2000) Validation of a questionnaire measuring patient satisfaction with general practitioner services. Quality Health Care 9: 210-215.

8. Neale J (2009) Research methods for health and social care, UK Palgrave Macmillan pp: 336.

9. Drewery K, Yates L, Birchall A, Barnett D, Buckley N, et al. (2012) Sheffields nurse-led heart failiure clinic: The patients' opinion. Br J Cardiology 19: 180-183.

10. Williams S, Williams J, Tcherveniakov P, Milton R (2012) Impact of a thoracic nurse-led chest drain clinic on patient satisfaction. Interact cardiovasc Thorac Surg 14: 729-733.

11. Karthikeyan M, Dahlmanni NA, Gupta N, Vivian AJ (2007) The cataract service satisfaction tool. Clin Governance: An Int J 12: 249-259.

12. Mussard J, Ashley FA, Newton JT, Kendall N, Crayford TJB (2008) What do you think of your dentist? A dental practice assessment questionnaire. J Evaluat Clin Pract 14: 181-184. 
Citation: Cock KA (2018) A Service Evaluation Project Comparing Telephone and Face to Face Colorectal 2 Week Wait Clinics. Adv Practice Nurs 3: 147. doi:10.4172/2573-0347.1000147

Page 7 of 7

13. Kelley K (2003) Good practice in the conduct and reporting of survey research. Int J Qual Health Care 15: 261-266.

14. Leon AC, Davis LL, Kraemer HC (2011) The role and interpretation of pilot studies in clinical research. J Psychiatr Res 45: 626-629.

15. Agresti A (2007) An introduction to categorical data analysis. (2 $2^{\text {nd }}$ edn) Wiley-interscience, USA.

16. Braun V, Clarke V (2008) Using thematic analysis in psychology. Qual Res Psychology 3:77-101.

17. Campbell J, German L, Lane C (1999) Radiotherapy outpatient review: A nurse-led clinic. Clin Oncol 13: 39-44.
18. Connor A, Mortimer F, Higgins R (2011) The follow-up of renal transplant recipients by telephone consultation: Three year experience from a single UK renal unit. Clin Med 11: 242-246.

19. Casey RG, Powell L, Braithwaite M, Booth CM, Sizer B, et al. (2017) Nurse-led phone call follow-up clinics are effective for patients with prostate cancer. J Patient Exp 4: 114-120.

20. Phend C (2009) Colonoscopy riskier in old age. Gastroenterol.

21. Sibbald B, Roland M (2017) Understanding controlled trials: Why are randomised controlled trails important? BMJ 316: 201. 